\title{
Nevirapine- versus Efavirenz-based antiretroviral therapy regimens in antiretroviral-naive patients with HIV and Tuberculosis infections in India: a multi-centre study
}

\author{
Sanjeev Sinha ${ }^{* *}$, Kartik Gupta', Srikanth Tripathy², Sahajal Dhooria', Sanjay Ranjan ${ }^{1}$ and R. M. Pandey ${ }^{3}$
}

\begin{abstract}
Background: According to World Health Organization (WHO) guidelines, which have also been adopted by the National AIDS Control Organization (NACO), India, Efavirenz-based Anti-Retroviral Therapy (ART) is better in HumanImmunodeficiency-Virus (HIV)-infected patients who are also being treated with Rifampicin-based Anti-Tuberculous Therapy (ATT). However, Efavirenz is much more expensive. We hypothesize that Nevirapine is a cheaper alternative that possesses equal efficacy as Efavirenz in HIV-Tuberculosis (TB) co-infected patients.

Methods: A parallel open-label randomized clinical trial was conducted at All India Institute of Medical Sciences (AllMS), New Delhi and National AIDS Research Institute (NARI), Pune. Those who were ART-naïve and co-infected with TB were randomized to receive either Nevirapine (Group 1)- or Efavirenz (Group 2)-based ART along with Rifampicin-based ATT. ATT was begun first in ART-nairve patients according to the NACO guidelines, with a median of 27 days between ATT and ART in both groups. The primary endpoint was a composite unfavourable outcome (death and/or ART failure) at 96 weeks, and the secondary outcome was successful TB treatment at 48 weeks.

Results: A total of 284 patients (mean age $36.7 \pm 8.1$ years) were randomized in a 1:1 ratio to receive either Nevirapine ( $n=144$ )- or Efavirenz ( $n=140$ )-based ART after a median ATT-ART gap of 27 days. The median CD4 count was 105 cells/ $\mu \mathrm{l}$, with a median viral load of 820,200 copies/ $\mu$ and no significant difference between the groups. Composite unfavourable outcomes were reported in 49 patients in the Nevirapine group and 51 patients in the Efavirenz group (35. $3 \%$ vs. 36.9\%; hazard ratio, $0.95,95 \%$ confidence interval (Cl), 0.63,1.43, adjusted). There was no difference in successful TB treatment outcome between the groups ( $71.5 \%$ vs. $65.6 \%, 95 \% \mathrm{Cl}-3.8,17.9$, adjusted). The results were similar, showing no difference between the groups in the two centres of the study after adjusting for disease stage.

Conclusions: Composite unfavourable outcome in HIV-TB co-infected patients who were ART-naïve showed no statistically significant difference in the Nevirapine or Efavirenz groups.. Therefore, Nevirapine-based ART is a reasonable alternative to Efavirenz in resource-limited settings. However, multi-centric studies with larger sample sizes are required to confirm these findings.
\end{abstract}

Trial registration: NCT01805258 (Retrospectively registered on March 6, 2013) Date of registration: March 2013.

Keywords: Antiretroviral, ART naïve, Efavirenz, Nevirapine, Tuberculosis, ATT

\footnotetext{
* Correspondence: drsanjeevsinha@gmail.com

'Departments of Medicine, All-India Institute of Medical Sciences (AllMS),

Ansari Nagar, New Delhi 110029, India

Full list of author information is available at the end of the article
} 


\section{Background}

Tuberculosis is the second most common opportunistic infection (after oral candidiasis) among newly diagnosed HIV-positive cases, with an incidence rate of approximately $10 \%$ [1]. India has the world's highest burden of Tuberculosis and the third largest number of people living with HIV in the world; it also ranks third in the world for HIV-associated TB [2]. In 2015, there were 0.1 million new cases of TB in PLHIV (people living with HIV) in India, with TB being responsible for approximately $15-18 \%$ of all deaths among PLHIV [3]. Globally, there were an estimated 1.1 million deaths due to HIV in 2015, including 0.4 million deaths due to TB co-infection. TB outcomes are worse in PLHIV due to high mycobacterial load, disseminated infection, drug interactions, and other factors. [4]. Rifampicin-based ATT is known to induce hepatic cytochromes, which cause decreased serum levels of many drugs, including those given in ART [5]. Co-management of HIV and TB is complicated further by Immune Reconstitution Inflammatory Syndrome (IRIS), pill burden, adherence and toxicity.

Nevirapine metabolism is more sensitive to induction of hepatic enzymes than that of Efavirenz; therefore, Efavirenz-based regimens are advocated as the first-line treatment in HIV-TB co-infected patients [6-9]. The impact of giving Rifampicin with Nevirapine on virologic outcome has yielded conflicting results [10-14]. The virologic response to Nevirapine-based ART in HIV-TB co-infection, when given at a standard dose, was comparable to that of patients not on concomitant ATT [15]. For HIV programs operating in countries such as India, fixed-dose combinations containing Efavirenz (Tenofovir-Lamivudine-Efavirenz) are less affordable than those containing Nevirapine [16]. India spent approximately 1.6 billion US \$ in 2015 towards HIV care and prevention strategies [16]. In 2012, only 50\% (44\%$58 \%)$ of PLHIV in India were receiving ART [17]. In the years to come, with improved diagnosis and treatment, we are likely to increase ART coverage, necessitating the availability of cheaper drugs with comparable efficacy.

Therefore, this study was conducted to measure outcomes in HIV-TB co-infected patients with Rifampicinbased ATT and either Nevirapine- or Efavirenz-based ART in ART-naïve patients.

\section{Methods}

A randomized, parallel design, open-label clinical trial was conducted at AIIMS, New Delhi, and NARI, Pune between September 2007 and December 2013. Eligibility criteria included ART-naïve patients, co-infected with $\mathrm{TB}$, who had not been started on ATT and were aged $>18$ years with no Diabetes Mellitus or co-infection with Hepatitis B or C. Patients taking anti-epileptic drugs, immunosuppressants or any other drugs that could induce liver microsomal enzyme systems were also excluded. All female participants were screened with a urine pregnancy test and were excluded if found to be pregnant since at the time of conducting the study, there were concerns regarding the safety of Efavirenz during pregnancy.

HIV infection was confirmed using a licensed ELISA kit. All patients underwent a physical examination, complete blood count, liver and kidney function testing, chest X-ray and abdominal ultrasonography (USG), along with tests for Hepatitis B Surface Antigen (HBsAg), anti-Hepatitis C Virus (HCV) antibodies, and Venereal Disease Research Laboratory (VDRL) testing. All HIV-positive patients were screened for TB. Pulmonary tuberculosis was diagnosed by sputum microscopy for Acid-Fast Bacilli (AFB) (two samples; on spot and earlymorning), chest X-ray and response to empirical ATT in sputum smear-negative patients with radiological or clinical findings suggestive of TB. Extra-pulmonary TB was diagnosed using radiographic, cytopathologic, histopathologic and biochemical assessments. ART-naïve patients coinfected with TB were randomized before the start of ATT in a 1:1 ratio to receive either Nevirapine- or Efavirenzbased ART with Rifampicin-based ATT.

ATT was given in the form of thrice-weekly therapy as DOTS (Directly Observed Therapy Short-course).

After ATT, ART was initiated, with a median ATTART gap of 27 days. ART was started after ATT to decrease the chances of IRIS. In the ART regimen, $\mathrm{Zi}$ dovudine, Lamivudine or Stavudine combined with either twice per day Nevirapine or once per day Efavirenz were given. ART doses were given according to NACO guidelines [18] (Zidovudine $300 \mathrm{mg}$ twice a day; Lamivudine $150 \mathrm{mg}$ twice a day and Stavudine $30 \mathrm{mg}$ twice a day), and Nevirapine was administered at a dose of $200 \mathrm{mg}$ a day for the initial fourteen days and was then escalated to $200 \mathrm{mg}$ twice a day. Efavirenz was given at a dose of $600 \mathrm{mg}$ once a day. All patients were given CoTrimoxazole prophylaxis according to NACO guidelines along with Pyridoxine $20 \mathrm{mg}$ once per day during ATT. No types of food were prohibited during the study period.

Patients were assessed at day 14 after the start of ART and then on day 28 , followed by every four weeks, for a total of 96 weeks at the ART centre.

CD4 counts were taken at 0,6 and 24 months by flow cytometry (BD FACS CALIBUR). Viral load was similarly measured at 0, 6 and 24 months using the AMPLICOR HIV-1 Monitor test, manufactured by ROCHE Diagnostics, and Abbott's real-time HIV-1 qualitative assay. Routine investigations (complete blood counts and kidney and liver function tests) were performed at $0,3,6,12,18$ and 24 months. Patients were enrolled after due consent was taken in their vernacular language. The protocol was approved by the ethics committees of the respective institutes. 


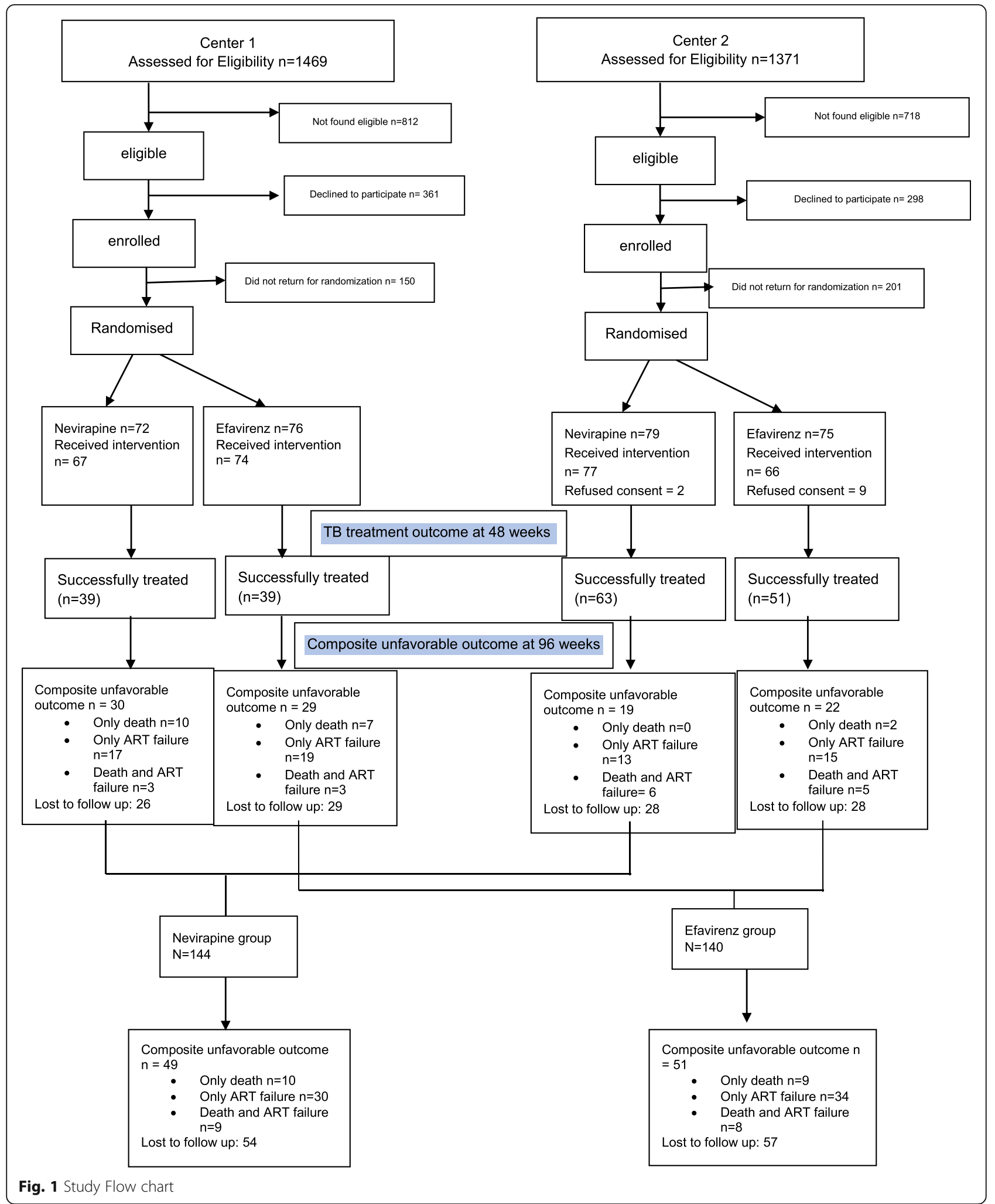

Clinical failure was defined as a new or recurrent WHO Stage 4 condition after at least 24 weeks of ART. Immunological failure was defined as a decrease in CD4 count from the baseline values; either a $50 \%$ decrease from the peak CD4 count during the treatment or persistent counts below 100 cells/ $\mu$ l after 24 weeks of 
Table 1 Baseline characteristics of the study population

\begin{tabular}{|c|c|c|c|}
\hline Variable & $\begin{array}{l}\text { Nevirapine } \\
n=144\end{array}$ & $\begin{array}{l}\text { Efavirenz } \\
n=140\end{array}$ & $P$ value \\
\hline Age, years: Mean \pm SD & $36.7 \pm 8.7$ & $36.7 \pm 7.6$ & \\
\hline \multicolumn{4}{|l|}{ Gender, number (\%) } \\
\hline Male & $104(72.2 \%)$ & $111(79.3 \%)$ & \\
\hline Female & $40(28.8 \%)$ & $29(20.7 \%)$ & \\
\hline $\mathrm{BMl}, \mathrm{Kg} / \mathrm{m} 2$ Mean $\pm \mathrm{SD}$ & $18.1 \pm 3.3$ & $18.5 \pm 3.3$ & \\
\hline CD4 count, cells/ul Median (Range) & $127(9-569)$ & $133(7-588)$ & 0.47 \\
\hline Viral load/ml Median (Range) & $334,225(120-5,000,000)$ & $173,000(230-5,800,000)$ & 0.17 \\
\hline \multicolumn{4}{|c|}{ WHO staging of HIV disease, number (\%) } \\
\hline Stage-1 & $4(2.9 \%)$ & $3(2.2 \%)$ & 0.03 \\
\hline Stage-3 & $39(27.8 \%)$ & $21(15.3 \%)$ & 0.04 \\
\hline Stage-4 & $97(69.3 \%)$ & $113(82.5 \%)$ & 0.04 \\
\hline \multicolumn{4}{|l|}{ Type of tuberculosis, number (\%) } \\
\hline PTB & $17(16 \%)$ & $24(20.7 \%)$ & 0.07 \\
\hline EPTB & $69(65 \%)$ & $82(70.7 \%)$ & \\
\hline Disseminated/Miliary TB & $20(19 \%)$ & $10(8.6 \%)$ & \\
\hline Category of ATT, number (\%) & & & 0.52 \\
\hline Category I & $124(86.8 \%)$ & $124(89.2 \%)$ & \\
\hline Category II & $19(13.3 \%)$ & $15(10.8 \%)$ & \\
\hline ATT-ART gap, days: Median (Range) & $27(-1$ to 100$)$ & 26 (4 to 96$)$ & 0.33 \\
\hline
\end{tabular}

treatment. Virologic failure was defined as viral load $>400$ copies/ $\mu \mathrm{l}$ after at least 24 weeks of ART. Combined ART failure was defined as the development of clinical, immunological or virologic failure at any time during the treatment. The composite unfavourable outcome was defined as either ART failure or death due to any cause during the 96-week follow-up period. Treatment outcomes of ATT were defined as per Revised National Tuberculosis Control Programme (RNTCP) guidelines, with successful treatment being defined as either completed treatment or cure.

The primary outcome of the study was to assess the proportion of subjects after 96 weeks who had a composite unfavourable outcome. The secondary outcome was an assessment of successful treatment of TB at 48 weeks.

A sample size of convenience was taken due to the paucity of both resources and time.

Block randomization with variable block size was used as a method of randomization to generate random numbers for allocation of patients into one of the two study groups. Codes were kept in an opaque envelope arranged serially, which was opened after the patient was found eligible for enrolment. These envelopes were kept with a person not involved in the study. The study flow chart is provided in Fig. 1.

The means and standard deviations (SD) were calculated for data with normal distribution, while medians and ranges were calculated for quantitative variables following non-normal distribution. The effect size of primary outcome at 96 weeks was computed using the difference in two proportions, and its 95\% confidence interval (CI) was determined. Time to primary outcome was analysed using the Kaplan-Meier survival analysis method, and overall survival curves between the two groups were compared using the log-rank test. All analyses were performed following the intention to treat principle. Continuous variables with normal distribution were computed using Student's t-test. Ordinal variables and variables following non-normal distribution were analysed using the Wilcoxon rank-sum test. Analysis of covariance was used to compute the effect size (95\% Confidence Interval), adjusting for stage of disease. The primary outcome analysis was the difference in the proportions of composite unfavourable outcomes at 96 weeks in the two groups, and the secondary outcome analysis was the comparison of successful TB treatment at 48 weeks. Statistical analysis was performed using STATA version 12.0 (STATA Corporation, College Station Road, Houston, Texas, USA).

\section{Results}

Of the 284 HIV-TB co-infected ART-naive patients enrolled, 144 were randomized to receive Nevirapine (group 1) or 140 Efavirenz (group 2). The baseline characteristics of the patients in the two groups are summarized in Table 1. 


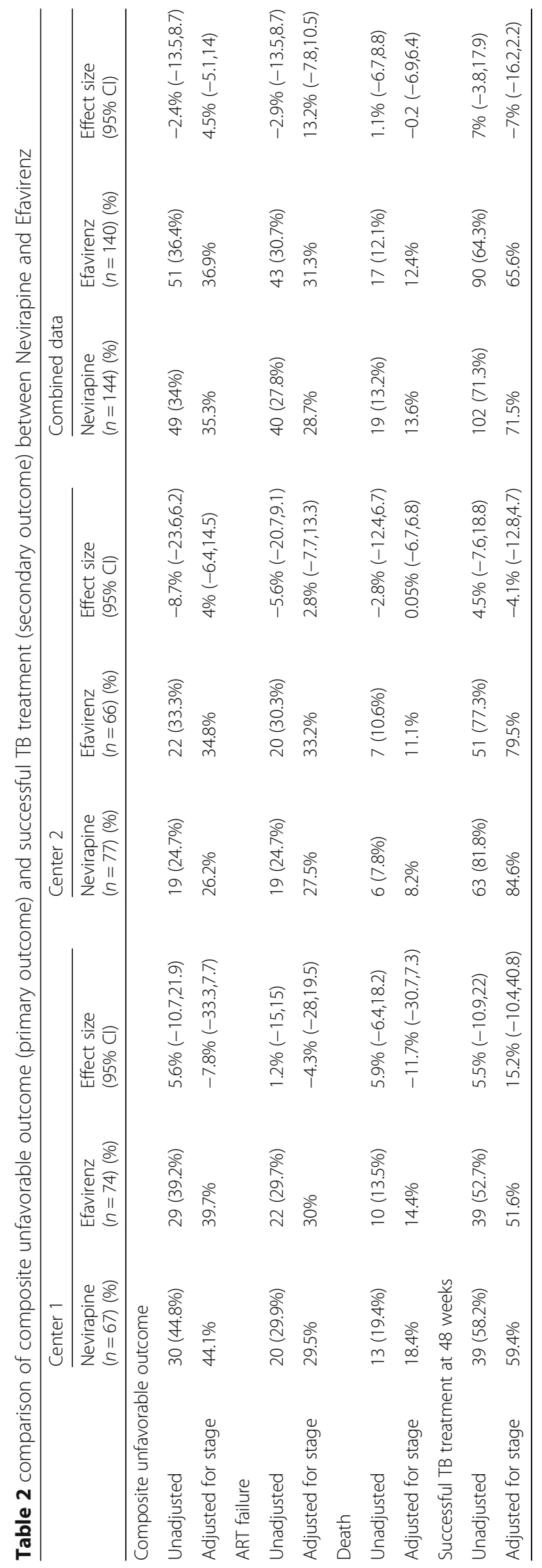




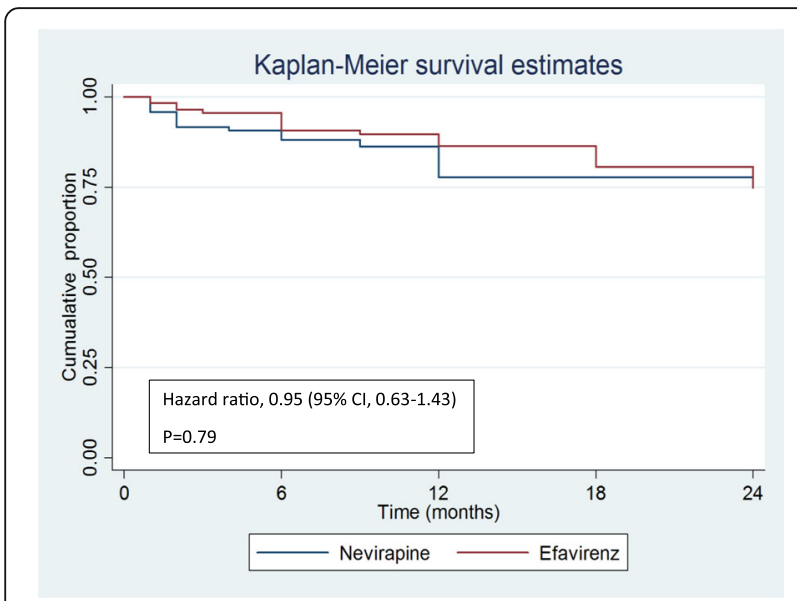

Fig. 2 Kaplan-Meier survival curve with cumulative probability of composite unfavorable outcome (death and or ART failure at 24 months)

The two groups were not significantly different except in WHO staging of disease, with significantly higher proportions of Stage 3 patients in the Nevirapine group and Stage 4 patients in the Efavirenz group. The 48week follow-up data were available for $127(88 \%)$ and 129 (92\%) patients in the Nevirapine and Efavirenz groups, respectively. The 96-week follow-up data were available for $103(71.3 \%)$ and $94(67.1 \%)$ patients in the Nevirapine and Efavirenz groups, respectively.

Composite unfavourable outcomes were reported in 49 patients in the Nevirapine group and 51 patients in the Efavirenz group ( $35.3 \%$ vs. $36.9 \%$; hazard ratio, 0.95 , 95\% Confidence Interval, $0.63,1.43 ; P=0.79$, adjusted) (Table 2 and Fig. 2). There was no significant difference in mortality between the two groups, even after adjusting for disease stage. There were no significant differences in the baseline CD4 counts and viral loads among patients who died in the 2 groups (Table 3). Of those who died, $60.7 \%$ participants $(78 \%$ in the Nevirapine group and 59\% in the Efavirenz group) had CD4 counts of less than 100 at baseline. Of the total 36 deaths, 28 (78\%) occurred during the ATT-ART overlap period.

Successful TB treatment outcome at 48 weeks (secondary outcome) was comparable between the two groups (71.5\% vs. $65.6 \%, 95 \%$ CI -16.2,2.2, adjusted) (Table 2). General metabolic parameters, such as haemoglobin, and measures of liver function, such as bilirubin, SGOT and SGPT, were comparable between the groups throughout the 96-week follow-up period. CD4 count (Fig. 3) and viral

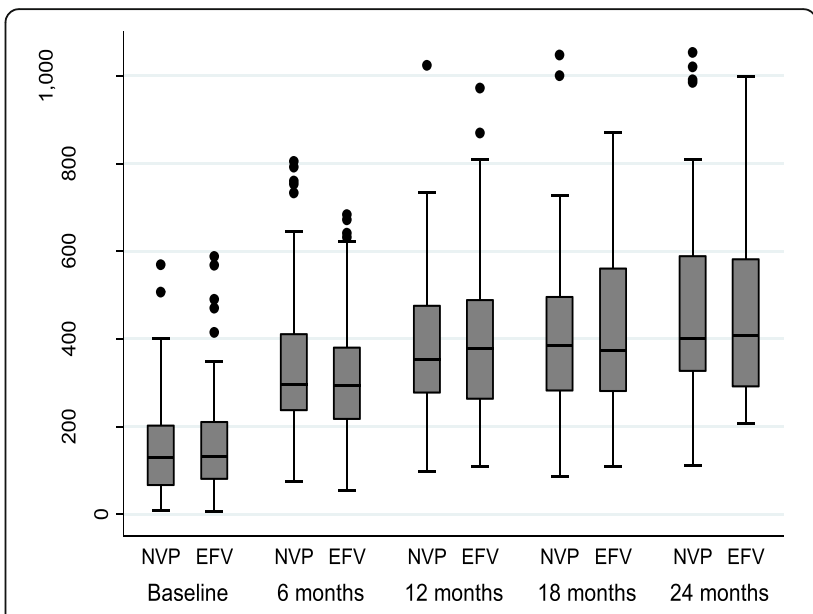

Fig. 3 CD4 cell count at follow-up intervals of 6 months in Nevirapine (NVP) and Efavirenz (EFV) group

load (Fig. 4) were not significantly different in the two groups throughout the follow-up period.

There were no significant differences in either the composite unfavourable outcome or the TB treatment outcome between the groups in the two study centres.

\section{Discussion}

Ruling out opportunistic infections (OIs) and treating the same, along with early initiation of ART, are the mainstays of HIV management. TB, being the second most common OI in PLHIV [1], causes significant morbidity and mortality because of the occurrence of disseminated infection, drug resistance, and other factors. This open-label, multicentre, randomized clinical trial showed that the overall outcomes were similar in the 2 groups, with similar rates of successful TB treatment outcome. Studies performed elsewhere have shown the benefit of Efavirenz over Nevirapine in TB co-infected participants, but the results of this study are contradictory, as there were similar mortality rates in the two groups. A total of $78 \%$ of all deaths occurred during the ATT-ART overlap period, thereby implying that ATT initiation and ART overlap are a crucial period for most of the participants and that once they pass this period, the chances of survival are improved.

Our results differ from the study performed by Swaminathan et al., which showed higher efficacy in the Efavirenz group vs the Nevirapine group at week 24 ( $85 \%$ vs $65 \%)$. However, in this study, participants were

Table 3 Baseline CD4 and viral load among patients who died

\begin{tabular}{llll} 
& Nevirapine $(n=19)$ & Efavirenz $(n=17)$ & $p$ value \\
\hline CD4 count Median Cells/ul (range) & $77(11-506)$ & $91(14-283)$ & 0.33 \\
Viral load Median Copies/ml (range) & $428,000(1498-1,760,000)$ & $113,027(583-3,105,916)$ & 0.09 \\
\hline
\end{tabular}




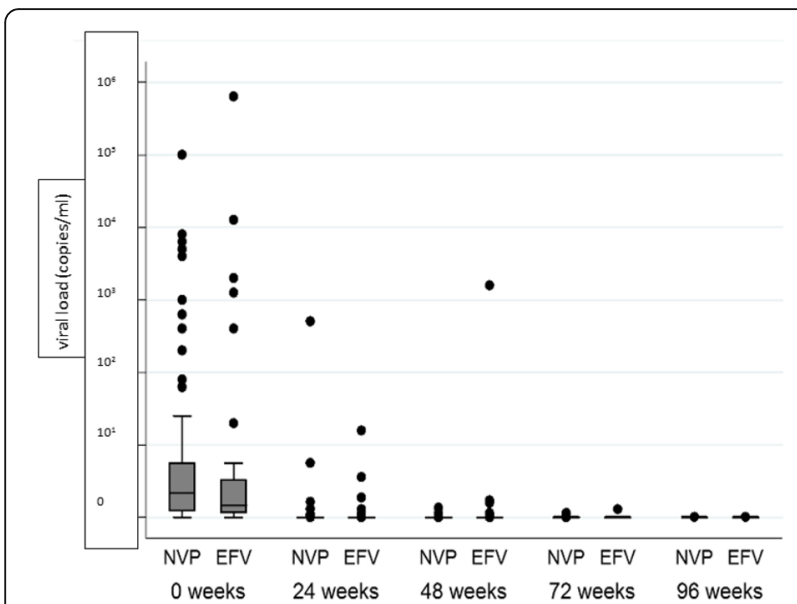

Fig. 4 Viral load at follow-up intervals of 6 months in log scale in Nevirapine (NVP) and Efavirenz (EFV) group

given once daily Nevirapine; furthermore, the study was a noninferiority trial, with fewer patients $(n=116)$.

The ATT outcomes were similar in both of the groups, even after adjusting for disease stage (a significantly higher proportion of participants in the Efavirenz group had Stage 4 disease, with a possibly higher degree of immunosuppression).

All participants were on DOTS therapy, and ART was started within a median of 27 days. With the introduction of daily Rifampicin-based ATT from 2016 under RNTCP, it remains to be seen how the interaction of ATT with ART therapy, especially Nevirapine-based therapy, will impact the clinical course. It is possible that the higher rate of failure in the Nevirapine group that was observed by Boulle et al. was because of this daily therapy.

IRIS was documented in a total of 8 patients (3\%) (6 in the Nevirapine group and 2 in the Efavirenz group). This finding is in contrast to the meta-analysis performed by Muller et al. [19], where the reported incidence was $15.7 \%$ (9.7-24.5). However, our findings are similar to the studies performed by Park et al. [20] and Lawn et al. [21], where the reported incidence was $2 \%$. The liver function tests (a marker for hepatotoxicity) were not significantly different between the two groups throughout the study period. The higher rate of hepatotoxicity with Nevirapine observed in the studies performed by Manosuthi et al. [22] and Van Leth et al. [23] might have been due to the inclusion of Hepatitis $B$ or Hepatitis C co-infected patients.

Efavirenz is metabolized through CYP2B6. Polymorphism in this enzyme complex has been implicated in Efavirenz-associated side effects, especially neurotoxicity. The prevalence of poor phenotypes of CYP2B6 has been reported to be as high as $20.56 \%$ in a study performed in similar population [24]. However, a study performed by
Ramachandran et al. [25] in South India among HIV-Tb co-infected patients showed that Rifampicin-based ATT did not significantly alter the pharmacodynamics of Efavirenz.

As discussed by De Nardo et al. [26], there are serious lapses and research gaps in identifying the ideal drug (Nevirapine or Efavirenz) in HIV-positive pregnant women to prevent mother-to-child transmission, especially in resource-limited settings. A similar deficiency has been noted in HIV-TB co-infected patients, the maximum proportion of which live in resource-limited countries with great financial strain and other infectious diseases with high mortality rates, such as malaria and childhood diarrhoea.

Randomization at the onset with a long-term followup of 96 weeks is the strength of our study. The TB treatment success rates in our study were consistent with the data from older studies showing cure rates between $59.4 \%$ to $97.1 \%$ [27].

The limits of our study include a high rate of loss to follow-up (approximately 30\%) and a sample size of convenience. With the introduction of daily Rifampicinbased ATT under RNTCP, DOTS with thrice-weekly ATT has been phased out; therefore, these results cannot be extended to the current treatment regimens for HIV-TB co-infection.

\section{Conclusion}

Nevirapine and Efavirenz were equally effective in terms of overall mortality and chances of ART failure. The TB treatment outcomes were similar between the two groups.

\section{Additional file}

Additional file 1: Workbook. (XLSX $21 \mathrm{~kb})$

\section{Abbreviations}

AlIMS: All-India Institute of Medical Sciences; ART: Anti-Retroviral Therapy; ATT: Anti-Tubercular Therapy; EFV: Efavirenz; HIV: Human Immunodeficiency Virus; NACO: National AIDS Control Organization; NARI: National AIDS Research Institute; NEV: Nevirapine; TB: Tuberculosis; WHO: World Health Organization

\section{Acknowledgements}

We thank the staff members of the ART clinic and DOTS for their help during patient enrolment and follow-up. We acknowledge the support and coordination of all staff of the NACO project and the Ph.D. students of the Department of Medicine and Microbiology.

\section{Funding}

National AIDS Control Organization (NACO), Ministry of Health and Family Welfare, Government of India.

Availability of data and materials

Yes, provided as a Additional file 1.

Authors' contributions

SS \& KG provided inputs to the study design, helped in data analysis and interpretation, wrote the manuscript, and did the final editing. SD, ST \& SR 
reviewed the literature and helped in interpreting data and writing the manuscript. RMP performed data analysis. All authors approved and read the final manuscript.

\section{Ethics approval and consent to participate}

This study has been approved by the Institute Ethics Committee, All-India Institute of Medical Sciences, New Delhi, India. Before the start of the study, all participants provided written informed consent. Participants were informed that they had the right to discontinue their participation or withdraw from the study at any time.

\section{Consent for publication}

Consent was taken from both the ethics committee and the subjects at the time of enrolment.

\section{Competing interests}

The authors declare that they have no competing interests.

\section{Publisher's Note}

Springer Nature remains neutral with regard to jurisdictional claims in published maps and institutional affiliations.

\section{Author details}

'Departments of Medicine, All-India Institute of Medical Sciences (AlIMS), Ansari Nagar, New Delhi 110029, India. ${ }^{2}$ National AIDS Research Institute (NARI), Pune, India. ${ }^{3}$ Biostatistics, All-India Institute of Medical Sciences, Ansari Nagar, New Delhi 110029, India.

Received: 28 June 2017 Accepted: 27 November 2017

Published online: 11 December 2017

\section{References}

1. Low A, Gavriilidis G, Larke N, B-Lajoie MR, Drouin O, Stover J, Muhe L, Easterbrook P. Incidence of opportunistic infections and the impact of antiretroviral therapy among HIV-infected adults in low- and middle-income countries: a systematic review and meta-analysis. Clin Infect Dis. 2016;62(12): 1595-603. doi: 10.1093/cid/ciw125.

2. World Health Organization: Global tuberculosis report 2016. Geneva: WHO 2016. Available: http://www.who.int/tb/publications/global_report/ gtbr2016_executive_summary.pdf

3. National AIDS Control Organisation India. Department of AIDSControl, Ministry of Health \& Family Welfare: Annual Report 2015-16. New Delhi: National AIDS control organization; 2015. Available: http://naco.gov.in/sites/ default/files/Annual\%20Report\%202015-16_NACO.pdf

4. De Cock KM, Soro B, Coulibaly I, Lucas SB. Tuberculosis and HIV infection in sub-Saharan Africa. JAMA. 1992;268(12):1581-7.

5. Niemi M, Backman JT, Fromm MF, Neuvonen PJ, Kivistö KT. Pharmacokinetic interactions with Rifampicin. Clin Pharmacokinet. 2003;42(9):819-50.

6. Manosuthi W, Sungkanuparph S, Thakkinstian A, et al. PlasmaNevirapine levels and 24-week efficacy in HIV-infected patients receiving Nevirapinebased highly active antiretroviral therapy with or without Rifampicin. Clin Infect Dis. 2006:43:253-5.

7. Cohen K, van Cutsem G, Boulle A, et al. Effect of Rifampicin-based antitubercular therapy on Nevirapine plasma concentrations inSouth African adults with HIVassociated tuberculosis. J Antimicrob Chemother. 2008;61:389-93.

8. Cohen K, Grant A, Dandara C, et al. Effect of Rifampicin-based antitubercular therapy and the cytochrome P450 2B6 516G>Tpolymorphism on Efavirenz concentrations in adults in SouthAfrica. Antivir Ther. 2009:14:687-95.

9. Gengiah TN, Holford NH, Botha JH, et al. The influence of tuberculosis treatment on Efavirenz clearance in patients co-infected with HIV and tuberculosis. Eur J ClinPharmacol. 2012;68:689-95.

10. Sinha S, Raghunandan P, Chandrashekhar R, Sharma SK, Kumar S, Dhooria S, et al. Nevirapine versus Efavirenz-based antiretroviral therapy regimens in antiretroviral-naive patients with HIV and tuberculosis infections in India: a pilot study. BMC Infect Dis. 2013;13(1):482

11. Swaminathan $S$, Padmapriyadarsini $C$, Venkatesan $P$, et al. Efficacy and safety of once-daily Nevirapine- or Efavirenz-based antiretroviral therapy in HIV-associated tuberculosis: a randomized clinical trial. Clin Infect Dis. 2011;53:716-24.

12. Shipton LK, Wester CW, Stock S, Ndwapi N, Gaolathe T, Thior I, et al. Safety and efficacy of nevirapine- and efavirenz-based antiretroviral treatment in adults treated for TB-HIV co-infection in Botswana. Int J Tuberc Lung Dis. 2009:13(3):360-6.

13. Manosuthi W, Sungkanuparph $S$, Tantanathip $P$, et al. A randomized trial comparing plasma drug concentrations and efficacies between 2 nonnucleoside reverse transcriptase inhibitor-based regimens in HIVinfected patients receiving Rifampicin: the N2R study. Clin Infect Dis. 2009:48:1752-9.

14. Boulle A, van Cutsem G, Cohen K, et al. Outcomes of Nevirapine and Efavirenz-based antiretroviral therapy when coadministered with Rifampicinbased antitubercular therapy. JAMA. 2008;300:530-9.

15. Manosuthi W, Tantanathip P, Chimsuntorn S, Eampokarap B, Thongyen S, Nilkamhang S, et al. Treatment outcomes of patients co-infected with HIV and tuberculosis who received a Nevirapine-based antiretroviral regimen: a four-year prospective study. Int J Infect Dis. 2010;14:e1013-7.

16. Overcoming barriers to access: http://www.msf.org/en/article/high-prices-hinderaccess-newer-hiv-drugs-and-optimal-tests-ensure-treatment-working

17. GHO | By country | India - statistics summary (2002 - present) [Internet]. WHO. [cited 2017 Jun 14]. Available from: http://apps.who.int/gho/data/ node.country.country-IND

18. National AIDS Control Organisation India, Department of AIDS Control, Ministry of Health \& Family Welfare: National AIDS control organization: antiretroviral therapy guidelines for HIV-infected adolescents and adults; New Delhi: NACO; 2013. Available: http://naco.gov.in/sites/default/files/ Antiretroviral\%20Therapy\%20Guidelines\%20for\%20HIVInfected\%20Adults\%20and\%20Adolescents\%20May\%202013\%281\%29_0.pdf

19. Muller M, Wandel S, Colebunders R, Attia S, Furrer H, Egger M. Immune reconstitution inflammatory syndrome in patients starting antiretroviral therapy forHIV infection: a systematic review and meta-analysis. Lancet Infect Dis. 2010;10(4):251-61.

20. Park WB, Choe PG, Jo JH, et al. Tuberculosis manifested by immune reconstitution inflammatory syndrome during HAART. AIDS. 2007;21:875-7.

21. Lawn SD, Bekker LG, Myer L, Orrell C, Wood R. Cryptococcocal immune reconstitution disease: a major cause of early mortality in a south African antiretroviral program. AIDS. 2005;19:2050-2

22. Manosuthi W, Mankatitham W, Lueangniyomkul A, Chimsuntorn S, Sungkanuparph S. Standard-dose Efavirenz vs. standard-dose Nevirapine in antiretroviral regimens among HIV-1 and tuberculosis co-infected patients who received Rifampicin. HIV Med. 2008;9:294-9.

23. Van Leth F, Phanuphak P, Ruxrungtham K, Baraldi E, et al. Comparison of first-line antiretroviral therapy with regimens including Nevirapine, Efavirenz, or both drugs, plus stavudine and lamivudine: a randomized open-label trial, the 2NN study. Lancet. 2004;363:1253-63.

24. Varshney E, Tandon M, Saha N, Ali S, Shrivastava V. Prevalence of poor and rapid metabolizers of drugs metabolized by CYP2B6 in North Indian population residing in Indian national capital territory. Springerplus. 2012; 1(1):34.

25. Ramachandran G, Kumar AKH, Rajasekaran S, Kumar P, Ramesh K, Anitha S, et al. CYP2B6 G516T polymorphism but not Rifampin Coadministration influences steady-state pharmacokinetics of Efavirenz in human immunodeficiency virus-infected patients in South India. Antimicrob Agents Chemother. 2009;53(3):863-8.

26. Pasquale De Nardo, Elisa Gentilotti, Boniface Nguhuni, Francesco Vairo et al. Efavirenz-based antiretroviral therapy versus nevirapine-including regimens for prevention of mother-to-child transmission of HIV option B plus in resource-limited settings: is there anything missing?, Expert Rev Anti-Infect Ther, 14:1, 19-27, DOl: https://doi.org/10.1586/14787210.2016.1116383.

27. El-Sadr WM, Perlman DC, Denning E, Matts JP, Cohn DL. A review of efficacy studies of 6-month short-course therapy for tuberculosis among patients infected with human immunodeficiency virus: differences in study outcomes. Clin Infect Dis. 2001;32:623-32. 